\title{
Role of thoracic radiotherapy in small cell lung cancer
}

Well before the modern WHO histological classification of lung cancer it was accepted that inoperable cancers sometimes responded dramatically to radiotherapy, occasionally resulting in a cure.$^{12}$ It also became clear at much the same time that the pattern of response and relapse in small cell lung cancer was quite different from other types, with a more rapidly evolving pattern of metastatic disease and, most importantly, a greater degree of radiosensitivity. ${ }^{3}$

Before the advent of combination chemotherapy as standard treatment for small cell lung cancer, radiotherapy was widely used. Fletcher, in the first edition (1966) of a classic radiotherapy text, ${ }^{4}$ noted that "undifferentiated carcinoma responds to radiation better than squamous cell carcinoma." Indeed it was only 30 years ago (1962) that the MRC designed a prospectively randomised study ${ }^{5}$ to compare radiotherapy and surgical resection for limited small cell lung cancer, an unusually far sighted initiative at the time. The study was finally launched in 1964 and showed unequivocally, and for the first time, the superiority of non-surgical treatment. ${ }^{6}$

The rapid establishment of combination chemotherapy as the standard approach for both limited and extensive small cell lung cancer was largely the result of further MRC studies in the UK, together with supporting evidence from the USA and Europe, ${ }^{78}$ although the early promise of combination chemotherapy proved difficult to sustain in the long term..$^{9}$ Indeed the MRC randomised study itself was typical of these trials in that a reasonably active regimen (in this case, a combination of methotrexate, cyclophosphamide and CCNU) was employed as an adjuvant to thoracic radiotherapy in half of the patients, and randomly compared with the "control" group undergoing radiotherapy at the same dose but without the chemotherapy. Very encouraging early (one year) results were obtained, with the group receiving radiotherapy + chemotherapy showing both a lower rate of metastatic progression $(57 \% v 79 \%)$ and far better rate of survival (34\% $v 18 \%)$. Later results were, however, disappointing, the second report ${ }^{9}$ showing no long term advantage although the median survival had improved from 25 weeks (radiotherapy alone) to 43 weeks (radiotherapy + chemotherapy). Early work from our own group suggested in a similarly treated group of patients that tumour size (taken from computed tomographic and radiographic measurement) might be a useful prognostic indicator both for response and survival, ${ }^{10}$ with a cutoff at $30 \mathrm{~cm}^{2}$.

The promising early results from combination chemotherapy in small cell lung cancer led many groups to abandon thoracic irradiation as "illogical" in a disease which was frequently systemic, even at presentation, and led to heated debate in the mid 1980s as to the value of thoracic radiotherapy as "consolidation" in patients now routinely treated with aggressive combination chemotherapy. ${ }^{11}{ }^{12}$ As Cohen ${ }^{11}$ pointed out in marshalling arguments against thoracic radiotherapy, "combination chemotherapy can achieve similar therapeutic results... and generally produces less severe toxicity (than radiotherapy)... combined modality treatment may result in debilitating chronic toxicity, and long term chemotherapy toxicity is qualitatively and quantitatively less severe." He pointed out correctly that long term local control required a high dose of thoracic radiotherapy (greater than $50 \mathrm{~Gy}$ with conventional fractionation) despite the relative radiosen- sitivity of the lesion, and also that significant acute toxic effects occur with combined modality treatment. Apart from the acute side effects, patients treated with combined chemotherapy + radiotherapy are clearly at risk of long term respiratory impairment from pulmonary/mediastinal fibrosis, oesophagitis and oesophageal stricture and, occasionally, significant radiation myelopathy. In addition, in patients with breast cancer-a group with a large well documented cohort of survivors (unlike small cell lung cancer) for study-thoracic irradiation also appears to produce significant myocardial ischaemia. By analogy this might also represent a major hazard in the small number of long term survivors of small cell lung cancer since a substantial portion of the myocardium is inevitably irradiated.

How to resolve these difficult issues? Our own group (the London Lung Group based at University College, Middlesex, Royal Brompton, Guy's, and the London Chest Hospitals) and many others have, in the past 10 years, conducted studies to compare combination chemotherapy alone or with thoracic radiotherapy, and have attempted large scale clinical trials to demonstrate any survival advantage that might intuitively be expected to be marginal. In order to minimise radiation toxicity and to exploit the apparent radiosensitivity of small cell lung cancer we limited the dose to $\mathbf{4 0} \mathrm{Gy}$ (conventional fractionation) in our prospectively randomised study of 363 patients using what was then a standard alternating chemotherapy regimen. ${ }^{13}$ Although we saw a small delay in the timing of local intrathoracic recurrence with radiotherapy the overall results were disappointing and both the survival of the two arms and the overall probability of intrathoracic relapse were similar. Other studies published in the late $1980 \mathrm{~s}$, mostly smaller than ours, essentially supported this view although the increasing understanding of the biological variability within such studies led, during this same period, to a recognition that even studies of several hundred patients were most unlikely to be large enough to detect a small but potentially important difference. Even enthusiastic medical scientists who were keen to pursue multicentre trials with patient randomisation as a routine part of clinical practice would be unlikely to complete a study which would prove numerically to be sufficiently powerful.

This issue was substantially resolved by the development of meta-analysis as a well validated clinical tool. The principle of the meta-analysis or trial overview technique is essentially that clinical trials from quite separate study groups, often scattered around the globe, can be "stacked up" together, providing they address the same issue. Largely developed by Peto and colleagues in the UK, this technique has proved enormously influential in confirming systemic treatments as an essential part of adjuvant therapy for breast cancer ${ }^{14}$ and has now been applied in an ever increasing number of clinical situations both in cancer and other disciplines. Despite initial misgivings as to the appropriateness of the technique, there seems no alternative if we accept the statistical view that for reliability the essential feature of any clinical trial of a new technique must be the acquisition of very large numbers of patients, all of whom have been randomised to receive or not receive the treatment in question.

This approach has now been applied to the issue of thoracic radiotherapy in patients with small cell lung cancer treated by combination chemotherapy. In 1992 in an 
international collaboration Pignon et al collected data from 13 studies totalling 2140 patients with limited disease, and with a median follow up period for the surviving patients of 43 months. ${ }^{15}$ They noted that 16 randomised studies has been conducted in the previous 15 years with inconsistent results. The analysis was undertaken to assess which of three possible explanations might be appropriate: (1) that technical factors such as radiation dose, volume treated, and choice of drugs administered are so critical that differences between them might modify the effect of treatment; (2) that thoracic radiotherapy might have no effect at all on survival, the differences in the results simply arising from the effect of statistical variations around a mean zero effect; (3) that thoracic radiotherapy has a moderate effect on survival, but that the trials do not have sufficient statistical power to test the hypothesis.

The results were both interesting and in some respects surprising. There was no shortage of statistical "events" to study since, of the 2103 patients included in the analysis, 1862 had died within the study period-992 in patients treated with chemotherapy and 972 in the 1111 patients assigned to chemotherapy + radiotherapy. Although this was not a very substantial numerical difference, the major conclusion was that there was indeed a small but statistically significant advantage for patients treated with the chemotherapy + radiotherapy combination, with a final point estimate of benefit clearly on the "treatment better" side of the isoeffect line and with confidence limits which did not cross into the "no benefit" area. The pooled relative risk from the analysis was 0.82 , corresponding to an $18 \%$ reduction in the risk of death from disease.

A further interesting trend emerged in that subgroup analysis showed a clear correlation between the degree of benefit obtained from thoracic radiotherapy and the age of the patient treated. Younger patients had more to gain, with a relative reduction in the risk of death in younger patients (under 55 years) of 0.72 -that is, a $28 \%$ reduction from the addition of radiotherapy to combination chemotherapy. In these patients the three year survival rates were $9.2 \%$ with chemotherapy alone and a dramatically better $17 \cdot 4 \%$ with the chemotherapy + radiotherapy combination. In patients over the age of 70 years the rates were similar: $10 \cdot 2 \%$ and $8 \cdot 7 \%$ respectively.

Despite the relatively poor overall long term survival we have come to expect in patients with small cell lung cancer (even those with limited disease), the Pignon analysis showed that the contribution of thoracic radiotherapy was very substantial in patients who were destined to survive. As expected, the initial part of the survival curve fell steeply in both groups whether or not thoracic radiotherapy was given. After 18 months or so, however, the benefit of thoracic radiotherapy became more apparent so that, at two years and beyond, the addition of thoracic radiotherapy appears to add a more important increment of survival with, for example, a three year survival rate of $14.3 \%$ in the chemotherapy + radiotherapy group compared with an $8.9 \%$ survival in patients treated with chemotherapy alone.

One of the strengths of the Pignon analysis was that it was based on data from individual patients submitted by each trial group and avoided publication bias by including unpublished data. Regrettably some $16 \%$ of patients were lost to follow up after three years and a further criticism of the study-as the authors themselves recognised-was the failure to evaluate non-lethal toxicity of treatment, making it impossible to apply a "cost-benefit" or "tradeoff" analysis against the potential advantage in longevity.

It seems unlikely that we will ever gain a clearer view of the potential benefit from thoracic radiotherapy in patients with limited small cell lung cancer since many have already concluded that the advantages of radiotherapy are now clear cut. In a disease with such high mortality, potential long term disadvantages of treatment are unlikely to weigh heavily, given the degree of additional benefit, particularly in patients under the age of 55 years. Important further questions still remain, however. What, for example, is the optimal schedule of treatment? Do we need to push the radiotherapy dose still higher? Is there a real difference between "early" and "late" thoracic radiotherapy in terms of treatment benefit, as recently suggested by Murray and others ${ }^{16}$ in a most important randomised study?

There are certainly theoretical reasons to favour early radiotherapy and concurrent or alternating chemotherapy regimens ${ }^{17}$ and future studies need to address this issue. Recent data from NCI, Canada suggest a substantial benefit from the early use of radiotherapy-that is, given concurrently with the first course of chemotherapy. ${ }^{16}$ In these patients the median survival was 21.2 months compared with only 16 months for patients treated by "delayed" radiotherapy (given at week 15 with the final cycle of chemotherapy). It now seems clear that virtually all patients with limited small cell lung cancer should undergo thoracic radiotherapy as part of their primary treatment, provided they are fit enough to receive it

J S TOBIAS

Clinical Director and Consultant in Radiotherapy and Oncology, Meyerstein Institute for Clinical Oncology,
Middlesex Hospital, London W1N \&AA

Reprint requests to: Dr JS Tobias

1 Roswit B, Kaplan G, Jacobson HG. The superior vena cava obstruction syndrome in bronchogenic carcinoma. Radiology 1953;61:722-36.

2 Suit $\mathrm{H}$, Lindberg R, Fletcher GH. Prognostic significance and extent of tumor regression at completion of radiation therapy. Radiology 1965;84: $1100-7$.

3 Selawry O, Hansen H. Lung cancer. In: Holland JF, Frei E III, eds. Cancer medicine. 1st edn. Philadelphia: Lea and Febiger, 1973: $1473-518$

4 Fletcher GH. Textbook of radiotherapy. Philadelphia: Lea and Febiger, 1966:405-9.

5 Medical Research Council. Comparative trial of surgery and radiotherapy for the primary treatment of small cell carcinoma of the bronchus. Lancet 1966;ii:979-86.

6 Fox W, Scadding JG. Medical Research Council comparative trial of surgery and radiotherapy for primary treatment of small-celled or oatcelled carcinoma of bronchus: 10 year follow-up. Lancet 1973;ii:63-5.

7 Medical Research Council Lung Cancer Working Party. Radiotherapy alone or with chemotherapy in the treatment of small-cell carcinoma of alone or with chemotherapy in the

8 Mira JG. Small cell lung cancer. In: Horwich A, ed. Combined radiotherapy and chemotherapy in clinical oncology. London: Edward Arnold, 1992:78-101.

9 Medical Research Council Lung Cancer Working Party. Radiotherapy alone or with chemotherapy in the treatment of small-cell carcinoma of the lung: the results at 36 months. Br $\mathcal{F}$ Cancer 1981;44:611-7.

10 Harper PG, Souhami RL, Spiro SG, Geddes DM, Guimaraes M, Fearon $\mathrm{F}$, et al. Tumor size, response rate and prognosis in small cell carcinoma of the bronchus treated by combination chemotherapy. Cancer Treat Rep 1982;66:463-70.

11 Cohen $M H$. Is thoracic radiation therapy necessary for patients with limited-stage small cell lung cancer? No. Cancer Treat Rep 1983;67:217-21.

12 Byhardt RW, Cox JD. Is chest radiotherapy necessary in any or all patients with small cell carcinoma of the lung? Yes. Cancer Treat Rep 1983;67:209-15.

13 Souhami, RL, Geddes, DM, Spiro SG, Harper PG, Tobias JS, Mantell BS, et al. A controlled trial of radiotherapy in small cell lung cancer treated by combination chemotherapy. BMf 1984;288:1643-6.

14 Early Breast Cancer Trialists' Collaborative Group (EBCTCG). Treatment of early breast cancer. Vol. 1. Worldwide evidence 1985-1990: a systematic overview of all available randomised trials of adjuvant endocrine systematic overview of all available randomised trials of adjuvant

15 Pignon J-P, Arriagada R, Ihde DC, Johnson DH, Perry MC, Souhami RL, et al. A meta-analysis of thoracic radiotherapy for small-cell lung cancer. $N$ Engl $\mathcal{F}$ Med 1992;327:1618-24.

16 Murray N, Coy P, Pater JL, Hodson I, Arnold A, Zee BC, et al. The importance of timing for thoracic irradiation in the combined modality treatment of limited-stage small-cell lung cancer. $\mathcal{F}$ Clin Oncol 1993;11: 336-44.

17 Arriagada R, Pignon JP, Le Chevalier T. Thoracic radiotherapy in small cell lung cancer: rationale for timing and fractionation. Lung Cancer 1989;5:237-47. 